\title{
Maneuvering in Shallow and Confined Water
}

\author{
Marc Vantorre $^{\mathbf{1}}$, Katrien Eloot ${ }^{\mathbf{1}, 2}$, Guillaume Delefortrie ${ }^{2}$, Evert Lataire ${ }^{\mathbf{1}}$, \\ Maxim Candries ${ }^{1}$, and Jeroen Verwilligen ${ }^{2}$
}

${ }^{1}$ Ghent University, Ghent, Belgium

${ }^{2}$ Flanders Hydraulics Research, Antwerp, Belgium

\author{
1 Maneuvering in Shallow Water \\ 2 Bank Effects \\ 3 Effect of Muddy Bottoms \\ 4 Ship-Ship Interaction \\ Nomenclature \\ Glossary \\ References
}

\section{MANEUVERING IN SHALLOW WATER}

\subsection{Introduction}

A ship's maneuverability depends on the water depth $h$ of the navigation area in relation to the draft $T$ of the vessel. PIANC (1992) makes a rather arbitrary distinction between deep $(h / T>3.0)$, medium deep $(1.5<h / T<3.0)$, shallow $(1.2<h / T<1.5)$, and very shallow water $(h / T<1.2)$. The effect of depth restrictions is noticeable in medium deep water, is very significant in shallow water, and dominates the ship's behavior in very shallow water. In dredged channels giving access to maritime ports, the ship's under-keel clearance (UKC), defined as $(h-T) / T$ and expressed as a percentage of draft, typically takes values of 10-20\% in sheltered areas and 15-40\% in areas subject to waves and swell (PIANC, 2014), which implies that navigating in medium

Encyclopedia of Maritime and Offshore Engineering, online @ 2017 John Wiley \& Sons, Ltd. This article is @ 2017 John Wiley \& Sons, Ltd.

DOI: 10.1002/9781118476406.emoe006

Also published in the Encyclopedia of Maritime and Offshore Engineering (print edition) ISBN: 978-1-118-47635-2 deep, shallow, and very shallow water is a common practice in ports and their access channels. In such navigation areas, characterized by limited depth and width, sea-going vessels are often confronted with completely different environmental conditions compared to navigation at sea for which most ships are designed and optimized. Besides the effects of the restricted depth, a ship also has to deal with the vicinity of banks, the presence of other shipping traffic, currents, speed restrictions, and so on. In such situations, the advice of a pilot with thorough knowledge of the local situation is often required in order to guarantee a successful operation.

For inland vessels, on the other hand, waterways with limited depth and width can be considered as a natural habitat. According to the Dutch waterway guidelines (Rijkswaterstaat, 2011), the depth of an inland waterway for commercial navigation with normal profile must be at least 1.4 times the draft of the reference ship, to be reduced to 1.3 for waterways with a narrow or single-lane profile.

\subsection{Effect of limited water depth on standard maneuvers}

Water-depth limitations will change considerably the pressure distribution around a moving vessel and will mostly cause an increase of the hydrodynamic forces due to the ship's motion through the water. Besides an increase of the ship's resistance, water-depth restrictions in general result in a decrease of her maneuverability, manifesting itself in the results of standard maneuvers. However, most ships perform such maneuvers only during the trials, which are always executed in deep water. Information about trials in limited water depth is therefore mostly based on simulations or model tests. Full scale test results are very rare; the 
most famous exception is the Esso Osaka test program (Crane, 1979). In 1977, maneuvering trials were conducted with this 278,000 ton deadweight crude oil carrier in the Gulf of Mexico, at UKCs of $320 \%, 50 \%$, and $20 \%$ of draft.

Compared to deep water, the characteristic dimensions of the turning circle in general monotonically increase with decreasing water depth, as illustrated in Figure 1 (left). Apparently, the dependency of the maneuverability in the lower UKC range is very significant: a small decrease in UKC results in a huge increase of the turning circle dimensions. As a result, larger bend radii are required in shallow navigation channels. Figure 1 (left) also reveals a decreased drift angle in a steady turn compared to deep water, resulting in a narrower swept path and a relatively smaller decrease of the ship's forward speed in the bend.

Water depth also has an effect on the course-checking ability of a ship: in (very) shallow water, overshoot angles during zigzag tests are considerably smaller compared to the deep water case. In spite of this apparently beneficial effect (Figure 1, right), the trials have a much longer duration as the yaw rates are significantly lower in the case of shallow water.

However, maneuvering characteristics of certain ship types may deviate from this general pattern in medium deep water. In the case of the Esso Osaka at 50\% UKC, for instance, the advance is slightly smaller compared to deep water. Moreover, the overshoot angles observed during zigzag tests may increase in the medium water depth range. This behavior is generally observed for full ship forms. In exceptional cases, the turning circle may even become smaller with decreasing water depth; a ship with wide beam (small $L / B$ ) and small draft (small $T / B$ ) appears to turn easier
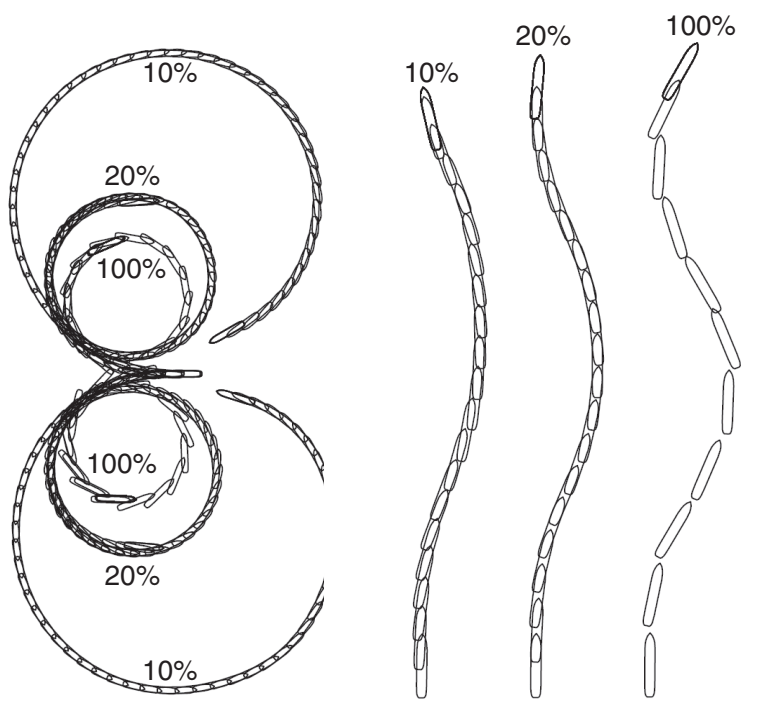

Figure 1. Turning circles and 20/20 zigzag tests with a ship model (confidential) at 10\%, 20\%, and $100 \%$ UKC, performed at BSHC, Varna, Bulgaria, on behalf of FHR, Antwerp, Belgium. in shallow than in deep water (Yasukawa and Kobayashi, 1995).

Water-depth limitations also influence the straight-line stability. While a UKC decrease initially may have an adverse effect on directional stability (as was observed in case of the Esso Osaka at 50\% UKC), in shallow water the dynamic stability increases with decreasing water depth.

Summarized, a ship's directional stability and maneuverability change considerably as a function of the available UKC. Especially in natural waterways (rivers, estuaries) where the water depth may vary significantly, both over the length of the channel and over the tidal cycle, a ship's maneuvering characteristics may be subjected to important changes during a transit through the channel.

\subsection{Effect of limited water depth on hull and rudder forces during maneuvering}

\subsubsection{Hull forces}

The dynamic equilibrium of a moving ship requires a balance between the inertial forces and moments and the hydrodynamic actions on the ship's hull, propeller(s), and rudder(s). For a maneuvering vessel, the kinematics and dynamics in the horizontal plane are of main concern, although the six degrees of freedom are not independent. This implies that the study of maneuvering focuses on lateral forces and yawing moments.

The lateral force $(Y)$ and yawing moment $(N)$ acting on a ship's hull caused by the hydrodynamic reaction to the relative motion of the ship with respect to the water can be considered as functions of the ship's velocity through the water in the horizontal plane, decomposed in a longitudinal $(u)$ and a lateral $(v)$ velocity component in a ship-fixed coordinate system and a yawing rate $(r)$, and the corresponding accelerations $\dot{u}, \dot{v}$, and $\dot{r}$. The most important acceleration-dependent force and moment, often denoted $Y_{\dot{v}} \dot{v}$ and $N_{\dot{r}} \dot{r}$, respectively, amplify the ship's mass inertia terms. $-Y_{\dot{v}}$ is referred to as the added mass for sway, $-N_{\dot{r}}$ as the added moment of inertia for yaw, see also article Maneuvering and Coursekeeping Characteristics. While in deep water, the added mass for sway is typically somewhat less than the ship's mass, its value may increase with a factor 4-5 in very shallow water, as illustrated in Figure 2. As a result, a ship's inertia increases significantly at reduced UKC, which results in a more sluggish behavior.

A similar trend is valid for the sway and yaw velocity-dependent forces and moments. Due to its shape in the horizontal plane, a ship hull can be interpreted as a lift-generating profile with chord $L$ and thickness $B$. The aspect ratio of this profile is very low: in deep water, the effective value can be considered as $2 T / L$, where the 

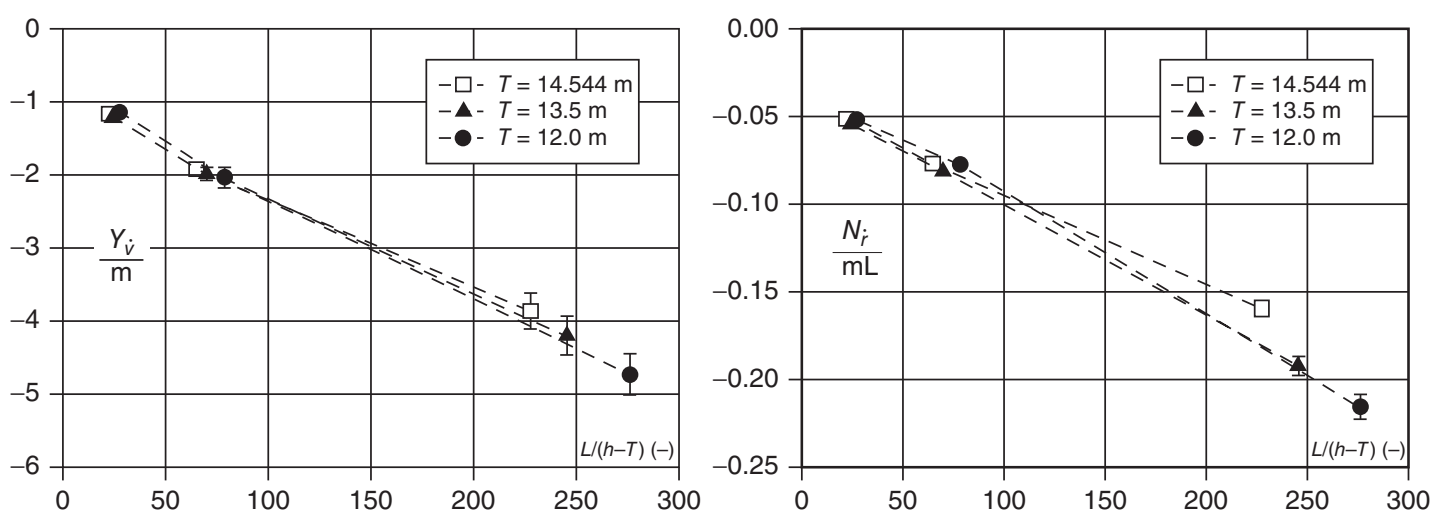

Figure 2. Nondimensional sway-added mass and yaw-added moment of inertia as a function of nondimensional UKC for a $1 / 80.8$ scale model of an 8000 TEU container carrier. (Reproduced with permission from Eloot, Vantorre, and Delefortrie, 2006. (C) International Marine Simulator Forum, 2006.)

factor 2 is due to the presence of the free water surface. With decreasing UKC, the cross-flow between both sides of the ship is increasingly obstructed, the effect of which is equivalent to an increase of the aspect ratio. In the limit case $h / T=1$, where no cross-flow is possible, the equivalent aspect ratio can be considered as infinite and the flow around the hull as two-dimensional.

As a result, the hydrodynamic lateral force and yawing moment acting on a ship moving at constant speed under a drift angle will increase significantly with decreasing UKC, as is illustrated in Figure 3.

Similarly, the yaw velocity-induced yawing moment gradually increases with decreasing UKC. A pure yaw motion also causes a hydrodynamic lateral force, which in deep water is practically negligible compared with the centrifugal inertia force $(-m u r)$. In shallow water, however, this hydrodynamic lateral force is increasingly important and is observed to counteract the centrifugal inertia force; the resultant of the hydrodynamic and inertial forces may even become centripetal at very low UKC.

\subsubsection{Control forces}

The forces on the rudder itself in general do not vary significantly with UKC. The flow toward the rudder is nevertheless affected by UKC limitations: the increased wake reduces the inflow, while on the other hand, the higher propeller loading increases the propeller-induced velocity. Both effects counteract each other, which almost results in a status-quo in most cases.

A rudder action induces an asymmetric flow that results in an asymmetric pressure distribution on both sides of the rudder and which eventually generates a lateral force on the rudder, which, expressed in the ship's coordinate system, is
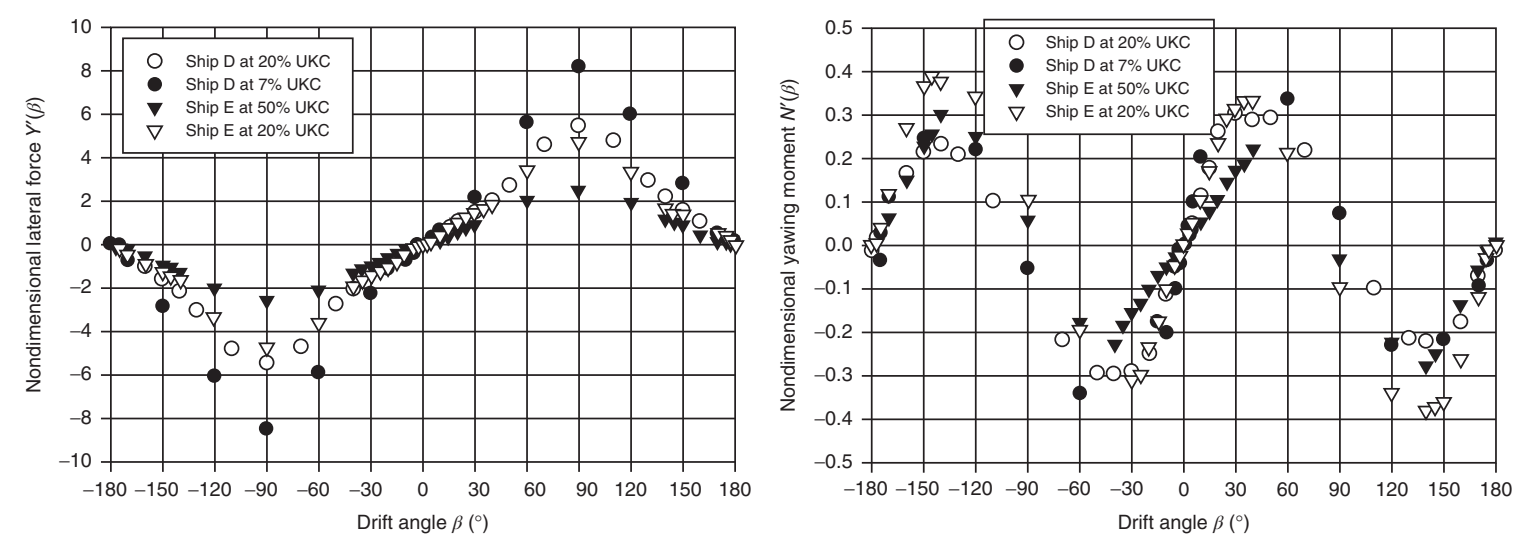

Figure 3. Nondimensional lateral force and yawing moment as a function of the drift angle $\beta=\tan ^{-1}\left(-v u^{-1}\right)$ for two ship models ( $D$ : container carrier and $E$ : tanker) at different UKC. (Reproduced with permission from Eloot, 2006. ( Katrien Eloot, 2006.)

Encyclopedia of Maritime and Offshore Engineering, online $\odot 2017$ John Wiley \& Sons, Ltc

This article is $\odot 2017$ John Wiley \& Sons, Ltd.

DOI: 10.1002/9781118476406.emoe006

Also published in the Encyclopedia of Maritime and Offshore Engineering (print edition) ISBN: 978-1-118-47635-2 
denoted $Y_{\mathrm{R}}$. The asymmetry of the pressure field is not only restricted to the rudder, but extends over the aft part of the ship's hull. Integration of the pressures induced by rudder action over the hull therefore results in a lateral force, which can be formulated as a fraction $a_{\mathrm{H}} Y_{\mathrm{R}}$ of the force on the rudder. In deep water, $a_{\mathrm{H}}$ is rather small, and the application point of the rudder-induced hull force is located near the ship's stern. With decreasing UKC, however, the magnitude of this force increases significantly; as its application point moves farther forward, the effect on the yawing moment is less important and can even have an adverse effect on the control actions when the rudder-induced hull force applies fore of midships.

\section{BANK EFFECTS}

\subsection{Introduction}

In restricted waters, a ship's behavior is affected by the lateral limits of the navigation area, such as banks and quay walls. These restrictions may influence the flow and pressure field around a ship and, therefore, the hydrodynamic forces and moments acting on the ship hull.

Different types of effects are distinguished, based on the relative motion of a ship with respect to the waterway boundary (ITTC, 2002):

- Bank effects are defined as the forces and moments acting on a ship due to a motion that has a mainly parallel orientation with respect to the bank.

- Cushion effects occur when a ship is moving laterally toward a solid boundary, typically resulting in an increase of the lateral hull force with decreasing bank clearance (e.g., berthing at a quay wall).

- Lateral restrictions influence a ship's frequency domain characteristics and, therefore, hydrodynamic memory effects occur in case of large accelerations or decelerations (e.g., contact of a berthing ship with fenders).

The following will concentrate on bank effects, which mainly occur when a ship is under way in a navigation area that is asymmetric with respect to the trajectory she is following. A ship navigating along the axis of a canal with a constant, symmetric cross-section will not experience any lateral force or yaw moment, but only an increase in resistance. If a ship is moving on an eccentric course, however, or if the navigation area is asymmetric, the flow around the ship will cause an asymmetric pressure field, resulting in a lateral force and a yawing moment.
In general, the relative water velocity at the side of the nearest bank will be increased compared to the open side. Due to Bernoulli's law, the pressure and, therefore, the water level will decrease more on the side of the nearest bank than on the open side. The resulting force will therefore (mostly) push the ship toward the nearest bank; for this reason this phenomenon is often called bank suction. As the water level depression is larger near the stern, while the bow wave may even result in an overpressure near the bow, this lateral force is accompanied by a yawing moment that moves the ship's bow away from the closest bank (bow-out moment). The vicinity of a bank also induces an increased ship resistance, as well as a modified squat and trim behavior.

\subsection{Parameters determining bank effects}

\subsubsection{Overview}

Ship-bank interaction forces and moments depend on several parameters (ITTC, 2002):

- Distance between Ship and Bank. In general, the interaction effects increase with decreasing bank clearance, although the yawing moment may in some cases decrease for very small clearances.

- Ship Speed. As bank effects are dominated by Bernoulli effects, they are generally proportional to the square of the ship speed, although in shallow water, forces and moments increase even more than quadratic.

- Water Depth to Draft Ratio. The ship-bank interaction yawing moment increases monotonically with decreasing UKC and becomes spectacularly large in very shallow water. The lateral force is directed toward the nearest bank in medium deep water and shallow water; however, tank tests with towed ship models have shown that in the very shallow water range, the water level between the ship's side and the nearest bank appears to rise, so that a repulsion from that bank occurs if $h / T$ is less than a critical value in the range 1.1-1.25.

- Propeller Action. The propeller-induced velocity modifies the pressure distribution near the stern, resulting in an additional attraction force between the stern and the bank, which reinforces the bow-out moment. At very low $h / T$, the bank repulsion effect observed for towed models mentioned above is changed into bank attraction for self-propelled models due to this effect.

- Bank Geometry. An arbitrary distinction can be made between vertical banks (quay walls), sloped (surface-piercing) banks, and submerged banks (e.g., dredged channels). 
Most of these parameters and their influence on bank effects are not independent from each other.

\subsubsection{Ship-bank distance and bank geometry}

While the distance of a ship's side to a vertical wall can be defined in an unequivocal way, it is less obvious how to define the distance to a sloped or submerged bank. For this reason, several authors have formulated expressions for an equivalent ship-bank distance or for the effect of bank geometry on the ship-bank interaction forces.

In the 1970s, with the arrival of very large crude carriers, Norrbin (1974) executed captive force measurements and free-response trajectory tests with a tanker model along different banks with the aim to develop an analytical formulation for the lateral force and yawing moment due to the presence of a vertical bank as functions of $h / T$ and a nondimensional ship-bank distance $\eta_{0}$ (Figure 4(a)). For sloping banks, a multiplication factor was formulated as a function of the slope factor $k$, as well as an attenuation factor $\mathrm{e}^{-2\left(h_{1}\right) /\left(h-h_{1}\right)}$ for flooded banks (Norrbin, 1985). The analytical models of Norrbin, although only based on one ship model, are often used in ship maneuvering simulation, thanks to their straightforward formulations and the easy determination of the bank distance parameter.

In canal sections, the banks at port and starboard sides have a counteracting effect; Ch'ng, Doctors, and Renilson (1993) extended Norrbin's research based on model tests with different ship types and developed generalized mathematical models, and introduced a nondimensional ship-bank distance parameter $y_{\mathrm{B} 3}$, based on the distances to each bank measured at half draft (Figure 4(b)):

$$
y_{\mathrm{B} 3}=\frac{B}{2}\left(\frac{1}{y_{\mathrm{p} 3}}+\frac{1}{y_{\mathrm{s} 3}}\right)
$$

In order to account for more complex, even arbitrary channel cross-sections, an equivalent nondimensional distance to bank (d2b) parameter was developed by Lataire and Vantorre (2008), based on a weight-distribution function $w(y, z)=\mathrm{e}^{-a|y|-b|z|}$ in the ship-bound coordinate system (see Figure 4(c)), which can be considered as an extension of the factor introduced by Norrbin (1985):

$$
\frac{1}{\mathrm{~d} 2 \mathrm{~b}}=\frac{\chi_{\text {ship }}}{2}\left(\frac{1}{\chi_{\mathrm{stb}}}-\frac{1}{\chi_{\text {port }}}\right)
$$

$\chi$ being the integral of the weight-distribution function over the area mentioned in the subscript: "ship" refers to the ship's cross-section, and "stb" and "port" to the part of the channel cross-section at starboard and port side, respectively, of the symmetry axis of the ship's cross-section.

\subsubsection{Ship speed, water depth, and bank geometry}

As already mentioned in Section 2.2.1, bank-induced lateral force and yawing moment are observed to increase more

(a)

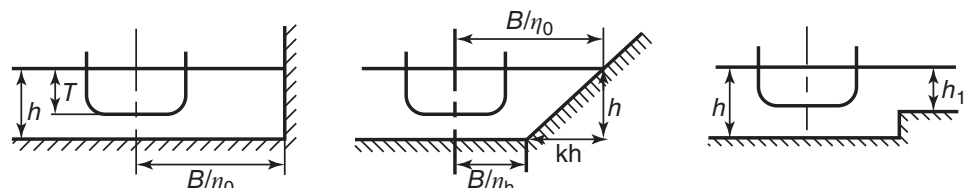

(b)
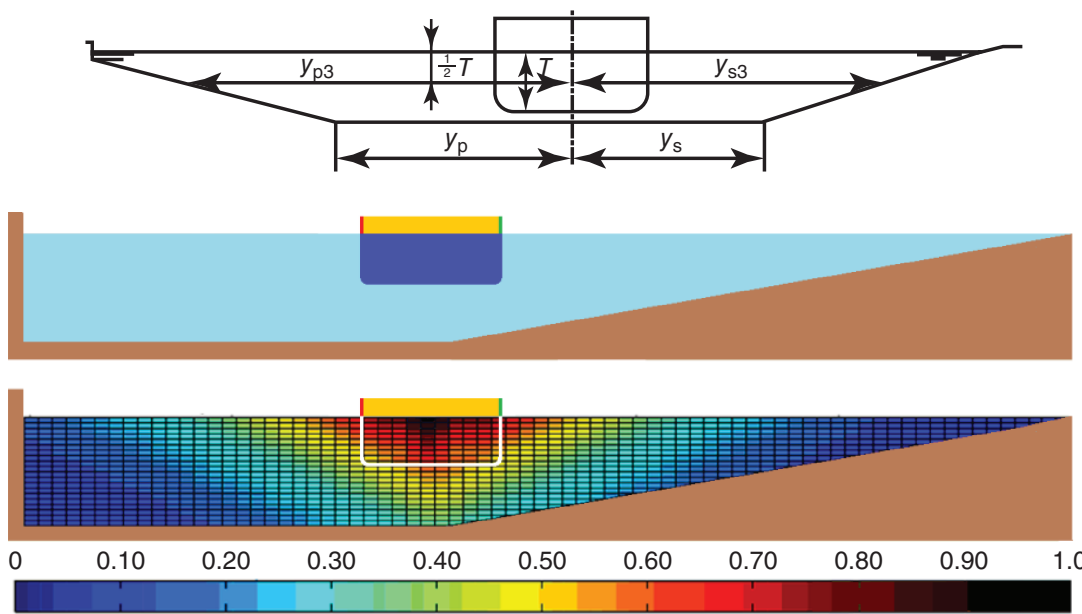

Figure 4. Ship-bank distance parameters. (a) Bank configurations considered by Norrbin (1974, 1985). (Reproduced from Norrbin, 1974. US Navy/Public Domain document.) (b) Definitions by Ch'ng, Doctors, and Renilson (1993). (Reproduced with permission from Ch'ng, Doctors, and Renilson, 1993. (C) IOS Press BV, 1993.) (c) Weight distribution function defined by Lataire and Vantorre (2008).

Encyclopedia of Maritime and Offshore Engineering, online ( 2017 John Wiley \& Sons, Ltd.

This article is @ 2017 John Wiley \& Sons, Ltd.

DOI: 10.1002/9781118476406.emoe006

Also published in the Encyclopedia of Maritime and Offshore Engineering (print edition) ISBN: 978-1-118-47635-2 
than proportional to the square of the ship's speed. On the basis of the comprehensive model-test series, Lataire (2014) concluded that bank effects are proportional to

$$
\frac{V^{2} / V_{\text {crit }}^{2}}{\sqrt{1-\left(V^{2} / V_{\text {crit }}^{2}\right)}}
$$

$V_{\text {crit }}$ is the critical speed of the ship in the waterway, which is a function of the blockage factor $m$ and the average water depth in the channel (Briggs et al., 2009):

$$
V_{\text {crit }}=\sqrt{g h_{\text {avg }}}\left(2 \sin \left(\frac{\operatorname{Arcsin}(1-m)}{3}\right)\right)^{3 / 2}
$$

The proportionality is valid for subcritical speeds up to about $0.84 V_{\text {crit }}$. As an alternative for the classical definition for the blockage factor $m$, that is, the ratio between the ship cross-section and the channel cross-section, an alternative equivalent blockage factor $m_{\mathrm{eq}}$ can be defined, accounting for the weight-distribution function introduced in Section 2.2.2. This allows account to be taken of the eccentric position of the ship in the channel, as well as arbitrary bank geometries.

\subsection{Controllability of a ship navigating parallel to a bank}

In order to maintain an eccentric lateral position in a channel, a rudder action directing the bow toward the closest bank is required to compensate the bank-induced yawing moment. Such a situation may occur in a two-way channel; prior to meeting, the vessels are lined up along their meeting lines. For a specific ship in a given loading condition with a specified UKC, the rudder angle required to compensate for bank-induced forces depends on the ship's speed, the applied propeller rate, and the ship-bank distance. For a specified engine setting, the required rudder capacity to counteract the bank effect can be calculated for each combination of speed and lateral position.

As an example, a typical panamax container carrier meeting a similar ship in the Gaillard Cut, the narrowest reach of the Panama Canal, is discussed. At this location, a ship preparing for a meeting will leave a clearance of about $1.5 B$ to the buoy line. Figure 5 illustrates the influence of the propeller rate on the controllability of the ship. For instance, the ship, sailing on her meeting line with a speed of 6.5 knots, requires $38 \%$ of the rudder capacity to counteract bank effects with propulsion slow, increasing to $70 \%$ at
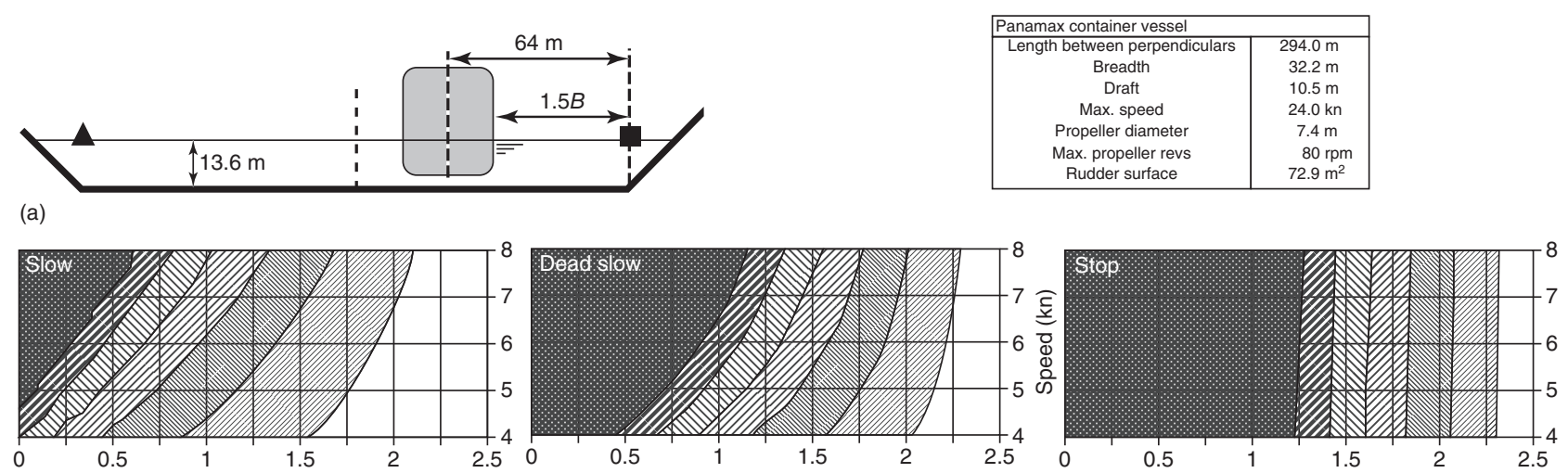

(b) Distance to buoy line/ship beam (-)

Distance to buoy line/ship beam (-)

Distance to buoy line/ship beam (-)
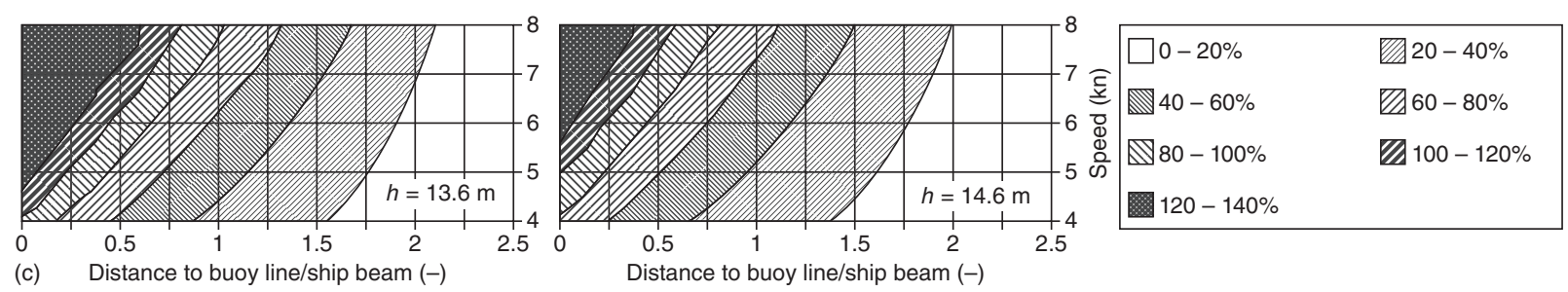

Figure 5. Panamax container vessel sailing on her meeting line in the Gaillard Cut. (a) Ship and canal geometry. (b) Required rudder capacity at different propeller rates as a function of ship speed and distance to buoy line. (c) Required rudder capacity: effect of canal deepening. (Reproduced with permission from Eloot, Verwilligen, and Vantorre, 2007. (c) Marc Vantorre, 2007.)

Encyclopedia of Maritime and Offshore Engineering, online @ 2017 John Wiley \& Sons, Ltd.

This article is @ 2017 John Wiley \& Sons, Ltd

DOI: 10.1002/9781118476406.emoe006

Also published in the Encyclopedia of Maritime and Offshore Engineering (print edition) ISBN: 978-1-118-47635-2 
dead slow, and $85 \%$ with the propeller stopped. A comparison is also made with an enhanced situation after channel deepening. For a ship with engine slow, the required rudder capacity drops from $38 \%$ to $25 \%$ if the depth is increased to $14.6 \mathrm{~m}$, due to the high sensitivity of the bank-induced forces with respect to UKC variations.

\section{EFFECT OF MUDDY BOTTOMS}

\subsection{Nautical bottom}

Due to sedimentation, permanent maintenance dredging works are required to keep many ports accessible for deep-drafted vessels. In case of hard bottoms (rock, clay, and sand), the depth can be measured unambiguously by means of echo-sounding techniques. If the bottom is covered with soft mud layers, however, the boundary between water and bottom may be hard to define, as the survey results will depend on the applied ultrasonic frequency: while high-frequency echoes (e.g., $210 \mathrm{kHz}$ ) reflect at the mud-water interface, lower frequency signals (e.g., $33 \mathrm{kHz})$ penetrate deeper into the mud. The difference between both signals may vary from a few decimeters to even $3-4 \mathrm{~m}$. While the upper part of this layer may be fluid (black water), the density and the rheological properties (viscosity and yield stress) of the layer gradually increase with depth.

In this case, the nautical bottom concept has to be introduced, defined by PIANC (1997) as the level where physical characteristics of the bottom reach a critical limit beyond which contact with a ship's keel causes either damage or unacceptable effects on controllability and maneuverability. The application range of this definition is not limited to muddy bottoms. In case of a hard bottom (e.g., rock), bottom contact will cause damage, while contact with a muddy bottom will rather result in unacceptable ship behavior.

The nautical bottom concept was introduced in the 1970-1980s in a few West-European harbors. It is a common practice to select a critical density as a criterion for the nautical bottom, typically around $1.2 \mathrm{ton} / \mathrm{m}^{3}$, because this characteristic can be measured in situ in a relatively easy and unambiguous way. However, the rheological behavior of fluid mud is not directly related to sediment density, but also depends on the mud composition. Eventually, mud rheology is more important than density, because it is the rheology that determines whether mud behaves like a fluid or like a solid material. Due to the complexity of mud rheology, it is up till now not feasible to use a rheology-related criterion for determining the nautical bottom, although several measuring systems are under development.

Maintenance-dredging problems in harbors have led to on-going research on mud sedimentology, and also on behavior of ships navigating with decreased or even negative UKC with respect to the mud-water interface (further denoted UKCi). Model test research was performed in the 1970s and 1980s at MARIN for investigating the access of deep-drafted tankers to the port of Rotterdam, at SOGREAH in the light of sedimentation problems in French harbors, and at Flanders Hydraulics Research (FHR, Antwerp). More recently, a comprehensive captive maneuvering program executed at FHR led to the development of mathematical maneuvering simulation models (Delefortrie, Vantorre, and Eloot, 2005) to determine new access criteria for the port of Zeebrugge. Besides model tests, full-scale experiments were conducted in Rotterdam, Nantes-Saint-Nazaire, and Zeebrugge in the 1970s and 1980s, and more recently in the port of Delfzijl (Verwilligen et al., 2014).

\subsection{Physical phenomena}

Ship behavior may be affected by the presence of mud due to two phenomena:

- the mud rheology, which is of particular importance if contact occurs between the mud layer and the ship's keel;

- the generation of undulations (internal waves) in the water-mud interface, which may not only affect the flow around a ship in contact with the mud layer, but also when a ship is moving with limited UKCi.

The internal wave pattern depends on the ship's forward speed. At very low speed, the interface remains practically undisturbed. At intermediate speed, an interface sinkage is observed under the ship's entrance, which at a certain section changes into an elevation. This internal hydraulic jump is perpendicular to the ship's longitudinal axis, and increases in magnitude while moving toward the stern with increasing speed (second speed range). At higher speeds, the interface jump occurs behind the stern (third speed range), with increasing angle between the ship's heading and the propagation direction of the jump (Figure 6).

The occurrence of these speed ranges can be explained by means of a simplified theory, assuming that both water and mud are ideal, inviscid fluids. For a ship moving at low speed above a mud layer, dynamic equilibrium leads to both a sinking and a rising of the water-mud interface, while only a sinking is feasible for speeds exceeding a critical value $U_{\text {crit }}$

Encyclopedia of Maritime and Offshore Engineering, online @ 2017 John Wiley \& Sons, Ltd.

This article is @ 2017 John Wiley \& Sons, Ltd.

DOI: 10.1002/9781118476406.emoe006

Also published in the Encyclopedia of Maritime and Offshore Engineering (print edition) ISBN: 978-1-118-47635-2 

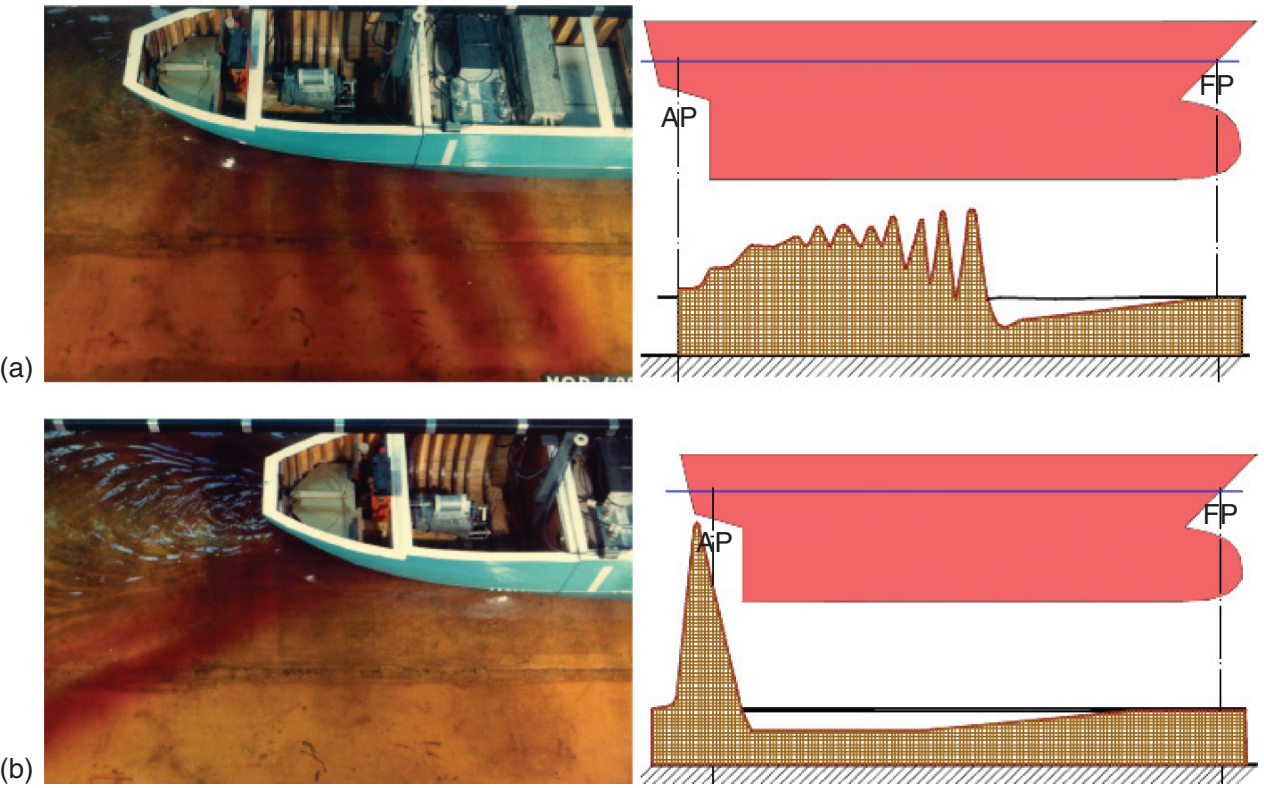

Figure 6. Mud-water interface undulations: second speed range (a) and third speed range (b). (Reproduced with permission from Vantorre, Laforce, and Delefortrie, 2006. ( Marc Vantorre, 2006.)

(Vantorre, 1991):

$$
U_{\text {crit }}=\sqrt{\frac{8}{27} g h_{1}\left(1-\frac{\rho_{1}}{\rho_{2}}\right)}
$$

$h_{1}$ being the depth of the water layer, and $\rho_{1}$ and $\rho_{2}$ denoting water and mud densities, respectively. Figure 7 shows that critical speeds are situated in the usual range at which harbor approach takes place.

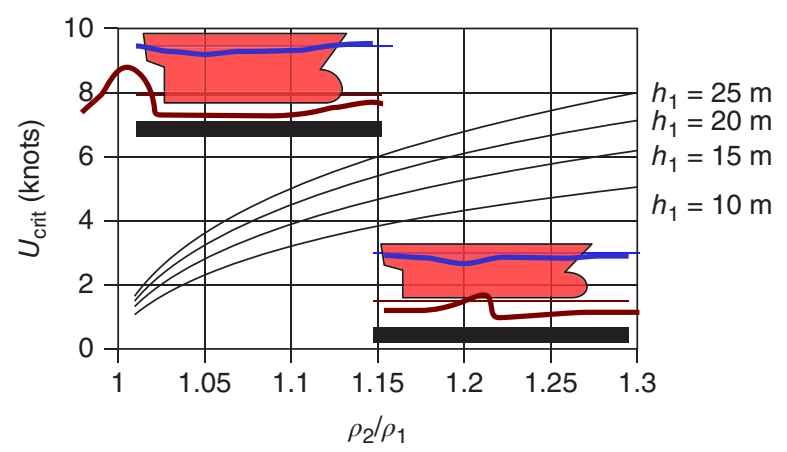

Figure 7. Critical speed separating second and third speed ranges as a function of mud-water density ratio for different water depths. (Reproduced with permission from Vantorre, Laforce, and Delefortrie, 2006. (C) Marc Vantorre, 2006.)

\subsection{Hydrodynamic forces on a maneuvering ship}

\subsubsection{Hull forces}

The following trends are of interest for understanding ship maneuverability in muddy areas:

- Hydrodynamic inertia terms increase significantly with decreasing water depth and increasing mud density and viscosity, see Figure 8. If the ship's keel penetrates deep into the mud, very large values are observed, but even when no contact occurs, the layer characteristics have an important effect. For a constant UKCi, the shallow-water effect is lessened with increasing layer thickness and decreasing mud density and viscosity. No abrupt transition is observed at zero UKCi.

- The drift-induced lateral force and yawing moment increase significantly with decreasing water depth. However, this increase appears to stagnate when the keel touches the interface. For a given positive UKCi, the presence of a mud layer appears to minimize the shallow-water effects, especially for layers with low density and viscosity. On the other hand, for a given UKC relative to the hard bottom, a mud layer always has an adverse effect.

- The yaw-induced lateral force follows the tendencies described in Section 1.3 for small and negative UKCi;

Encyclopedia of Maritime and Offshore Engineering, online @ 2017 John Wiley \& Sons, Ltd.

This article is @ 2017 John Wiley \& Sons, Ltd.

DOI: 10.1002/9781118476406.emoe006

Also published in the Encyclopedia of Maritime and Offshore Engineering (print edition) ISBN: 978-1-118-47635-2 


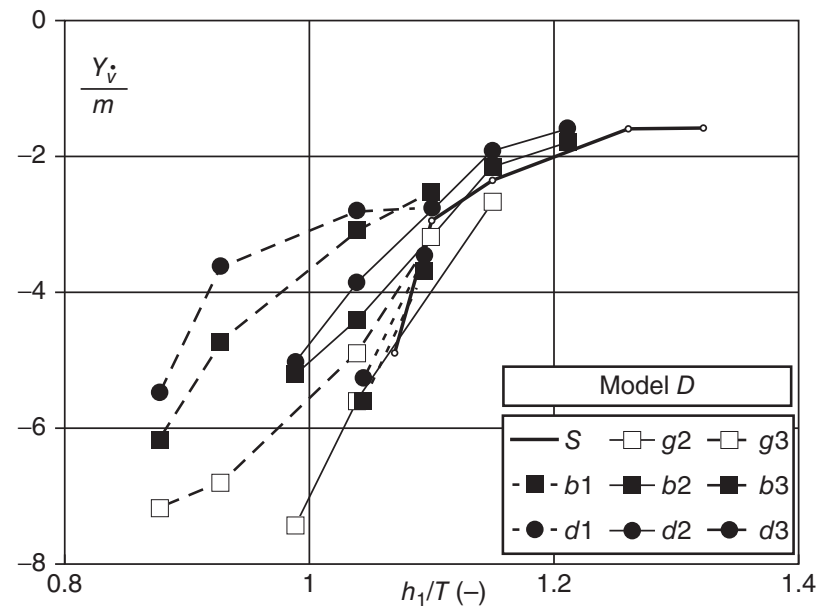

Figure 8. Effect of bottom characteristics and UKCi on the sway-added mass of a container carrier: $S=$ solid bottom; $g / b / c / d=$ mud density $1.25 / 1.18 / 1.15 / 1.10 \mathrm{ton} / \mathrm{m}^{3} ; 1 / 2 / 3$ : increasing layer thickness. (Reproduced with permission from Delefortrie, 2007. (C) Guillaume Delefortrie, 2007.)

the resulting lateral force even becomes centripetal. The transition from centrifugal to centripetal action takes place at a larger UKCi when the mud density and viscosity increase and the layer thickness decreases. Therefore, this effect is not typical for muddy areas, but should rather be considered as a (very) shallow water effect. Moreover, the yaw-induced yaw moment increases with increasing density and decreasing UKCi.

\subsubsection{Rudder forces}

The forces caused by rudder action depend on the axial flow into the rudder. This flow is a function of the forward speed, propeller rate, and rudder wake factor. The latter is significantly affected by the bottom condition and the UKCi: the wake factor decreases and, consequently, the flow to the rudder improves with increasing mud density and increasing UKCi. When the ship penetrates deep into soft, low density mud layers, however, the inflow to the rudder is affected unfavorably.

At near-zero UKCi values, due to interface undulations the ship's keel touches both water and mud, which may cause rudder instability: small rudder angles sometimes induce unexpected effects.

\subsubsection{Propeller-induced forces}

The longitudinal force acting on the ship due to propeller action depends on the propeller thrust, but also on the thrust-deduction factor. A larger value for the latter-which implies a smaller longitudinal force for a given thrust-is

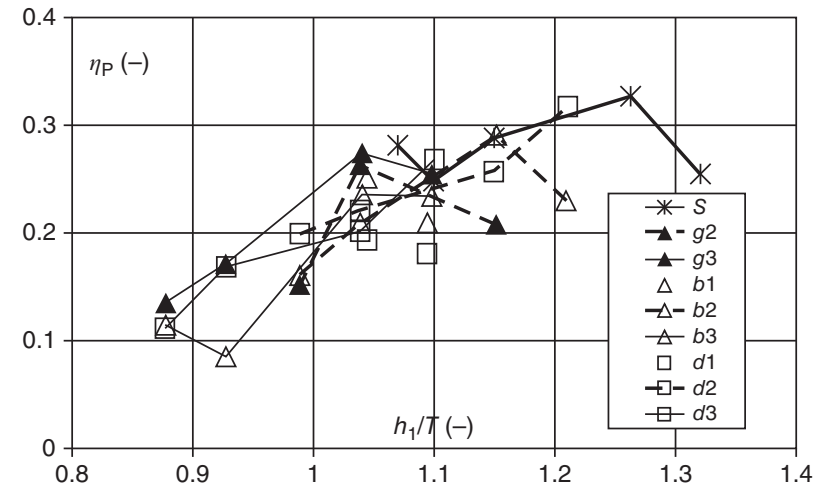

Figure 9. Overall propeller efficiency of a container carrier model: effect of bottom characteristics and UKCi. Symbols: see Figure 8. (Reproduced with permission from Delefortrie, 2007. (C) Guillaume Delefortrie, 2007.)

obtained at positive UKCi with high-density mud layers; at negative $\mathrm{UKCi}$, on the other hand, the thrust deduction factor is larger for the lowest densities.

The propeller thrust is determined by the propeller rate and the axial inflow velocity. The latter depends on the ship's forward speed, but also on the wake factor: a larger value for this factor implies a smaller inflow velocity and, therefore, a higher propeller loading. The wake factor is clearly affected by the bottom conditions: it increases when navigating above or through low-density mud layers, while a significant decrease is observed in contact with high-density mud layers.

Figure 9 shows the effect of mud on the overall propeller efficiency: compared to a solid bottom, a significant loss of efficiency is observed, especially at negative UKCi.

\subsection{Ship performance and maneuverability}

\subsubsection{Speed and propulsion}

The relationship between forward speed and propeller rate clearly illustrates the effect of interface undulations on a ship's propulsive performance. In the second speed range, as defined in Section 3.2, a given propeller rate results in a significantly lower speed above a muddy bottom; a similar effect was observed recently during full-scale observations (Verwilligen et al., 2014). An increased effort is required to reach the third speed range, where the mud effect practically disappears. The transition between both ranges is very clear at $10-20 \% \mathrm{UKCi}$, but is smoothened at negative UKCi.

This effect is not caused by increased resistance, but rather by obstruction of the flow to the propeller due to internal waves. Deeper penetration into mud layers, however, leads to a significant resistance increase. 


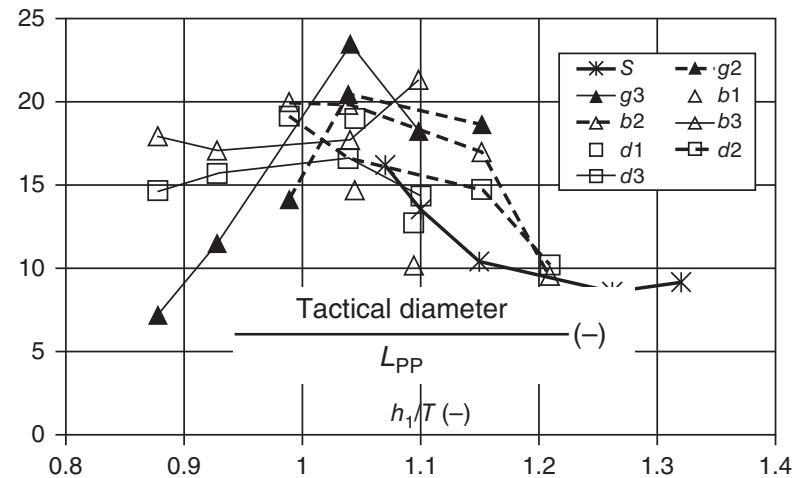

(a)

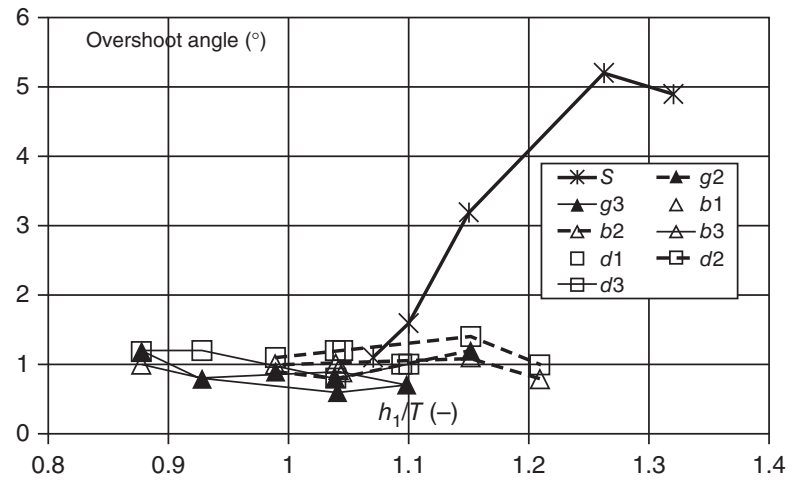

(b)

Figure 10. Tactical diameter (a) and first overshoot angle for 20/20 zigzag (b) for a container carrier model: effect of bottom characteristics and UKCi. (Reproduced with permission from Delefortrie, 2007. @ Guillaume Delefortrie, 2007.)

\subsubsection{Standard maneuvers}

Simulated turning circles show that a ship's turning ability generally decreases when a fluid mud layer is present. The tactical diameter appears to reach a maximum at a very small positive $\mathrm{UKCi}$, but decreases once the keel penetrates the mud layer and, in high density mud layers, even becomes smaller than above a solid bottom (Figure 10). The drift angles during turning-circle maneuvers are very small above and in mud.

With respect to zigzag tests, the first overshoot angle takes much smaller values above and in mud layers compared to a solid bottom condition, see Figure 10.

\subsubsection{Actual practice}

In several ports, the introduction of the nautical bottom concept has resulted in navigation with reduced UKCi; in case of mud layers with important thicknesses, even navigating through the mud layer is a common practice. In particular, in the port of Zeebrugge, a maximum penetration of $7 \%$ of draft into the mud layer is commonly accepted as a safe limit. Even larger penetration depths are applied in the port of Emden, where the mud is permanently fluidized.

Pilots and captains, however, have to account for a modified ship behavior, for example, by anticipating the increased inertia, strict speed limitations, and sufficient tug assistance. Not only the nautical bottom level has to be known, but also the position of the interface is of importance. In general, a slight negative UKCi results in a more stable and predictable behavior compared to a small positive UKC. Contact with consolidated mud layers, however, may lead to uncontrollable speed and heading, and should be avoided.

\section{SHIP-SHIP INTERACTION}

\subsection{Types of ship-ship interaction}

One of the problems interfering with navigation in restricted channels is the hydrodynamic interaction between ships. Four types of interaction will be considered:

- interaction between ships advancing at parallel courses: during overtaking, ships are sailing in the same direction, while encountering (meeting, reciprocal, or head-on passing) occurs with ships sailing in opposite directions;

- interaction with a moored ship;

- interaction between ships advancing at approximately equal forward speed in parallel and in close proximity to each other, which occurs during lightering and underway replenishment (UNREP) maneuvers;

- interaction of tugs with ships.

\subsection{Ships moving on parallel courses}

\subsubsection{Encountering}

In general terms, the interaction effects (Figure 11) begin to be felt when the bows of both ships are pushed away from each other, which is accompanied by a slight increase in speed. As the ships pass, the bow-out yaw moment turns to bow-in and the repulsion reduces. The bow-out moment then returns as passing continues but is now stronger and may cause the ships to sheer away from each other once they have passed. A reduction in speed may also be felt. Finally, a weak bow-in moment accompanied by a repulsion may 

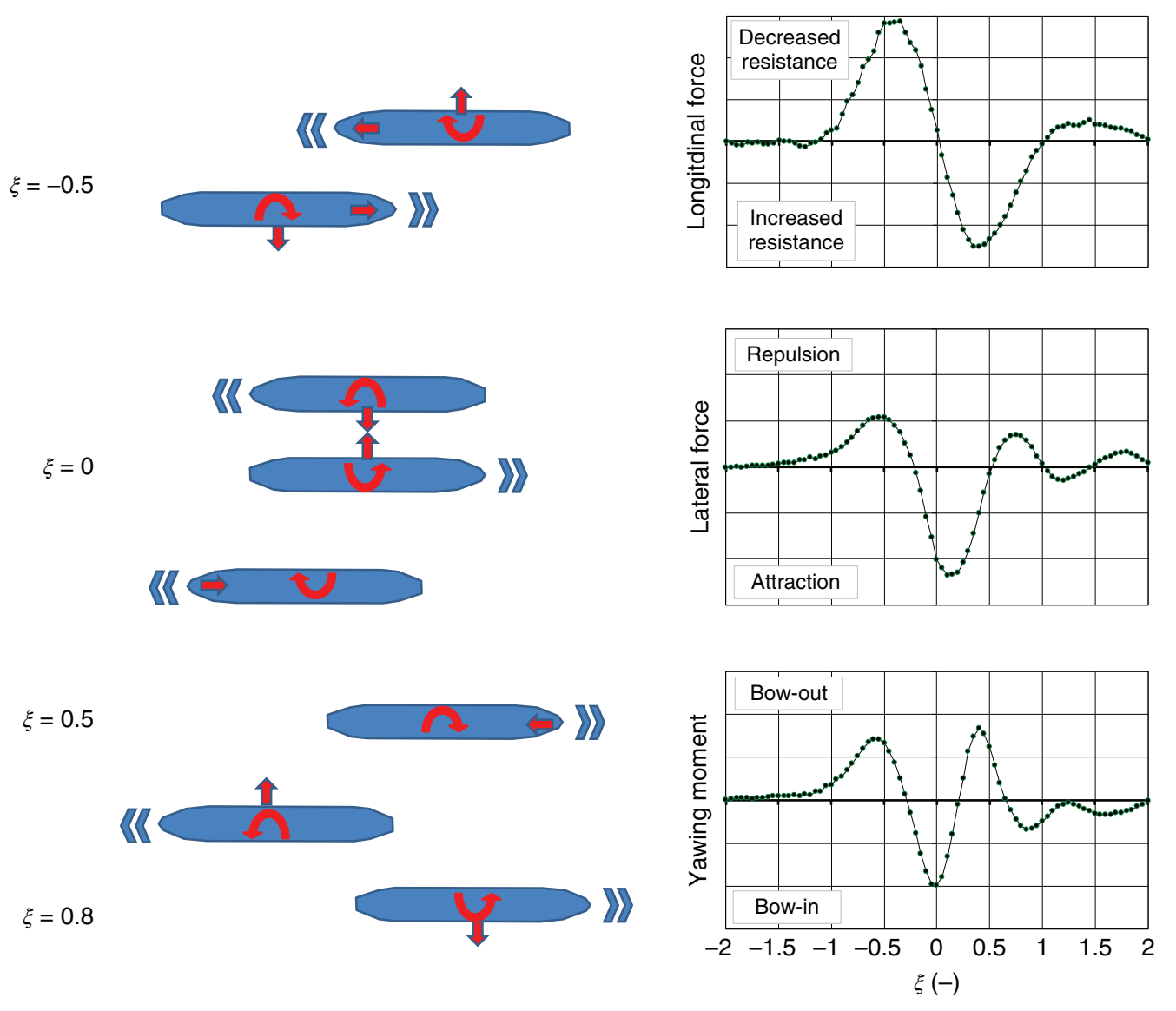

Figure 11. Graphical indication of the horizontal interaction effects during encountering maneuvers, $\xi$ being a nondimensional notation for the longitudinal separation between both midships sections (FHR).

be felt (Dand, 1995; Vantorre, Verzhbitskaya, and Laforce, 2002). Similarly, the varying pressure distributions around the ships affect the sinkage and trim of both vessels.

From an interaction point of view, passing on reciprocal courses has the merit of happening quickly so that the ship often does not have time to react to the various interaction forces and moments she feels. Usually, the dominant effects are the bow-out moments as the ships begin to pass and the stronger bow-out moments once passing is almost over.

\subsubsection{Overtaking}

Typical interaction forces are shown in Figure 12 for both ships involved. As the overtaking vessel overhauls the other vessel, a bow-in moment is first experienced by the fastest ship. The overtaken ship will then experience a strong bow-out moment followed by a bow-in moment. The sway force on the overtaken ship is characterized by a sequence of repulsion, attraction, and repulsion, comparable to encounter maneuvers. The overtaken ship first experiences a resistance increase but when the overtaking ship gets in front of the overtaken ship, a resistance decrease causes speeding up of the overtaken ship, whereas the overtaking ship slows down; this renders overtaking more difficult (trapping).

As the relative velocity during overtaking may be low, interaction has time to take effect. A collision scenario is shown in Figure 13a and is caused when the overtaken ship turns across the bows of the overtaking ship, which may perversely turn toward her. If a collision does not occur and the overtaking vessel moves past the other, both ships will feel powerful bow-out moments together with a mutual attraction. This may cause both ships to "fly apart" and their sterns to collide, as shown in Figure 13b (Dand, 1995).

\subsection{Interaction with moored ships}

Ships moored in harbors experience hydrodynamic forces due to other ships passing nearby, see Figure 14, as well as vertical motions. The passing vessel induces forces on the moored vessel that are associated with the low-frequency

Encyclopedia of Maritime and Offshore Engineering, online $\odot 2017$ John Wiley \& Sons, Ltd.

This article is @ 2017 John Wiley \& Sons, Ltd.

DOI: 10.1002/9781118476406.emoe006

Also published in the Encyclopedia of Maritime and Offshore Engineering (print edition) ISBN: 978-1-118-47635-2 

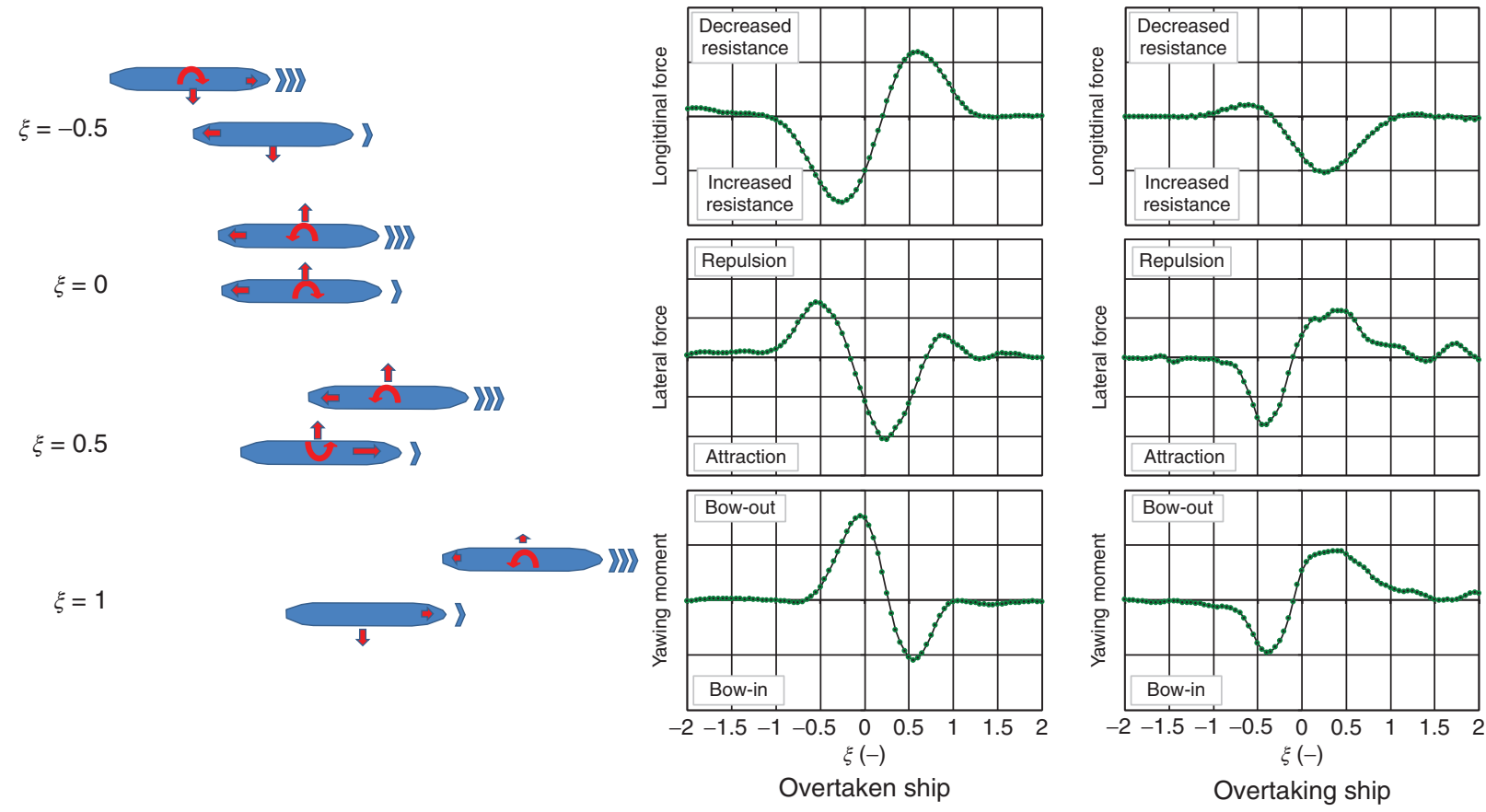

Figure 12. Graphical indication of the horizontal interaction effects during a typical overtaking maneuver (FHR).

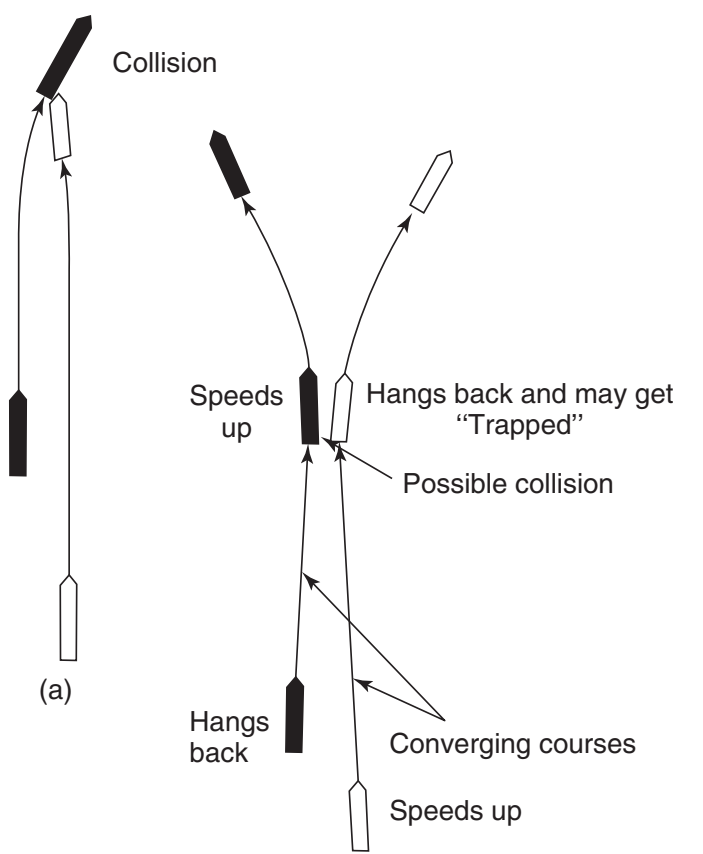

(b)

Figure 13. $(\mathrm{a}, \mathrm{b})$ Possible collision situations during an overtaking maneuver. (Reproduced with permission from Dand, 1995. (C) BMI Ltd, 1995.)

primary pressure system and forces that are associated with the higher-frequency secondary pressure system. The low-frequency (suction) forces are more significant for the moored ship at low speeds, while wash waves become important at near-critical or supercritical speeds (Pinkster, 2004; Van Der Molen et al., 2011).

The interaction forces induce motions of the moored ship that may hinder (un)loading operations or cause damage to the mooring system. Even though the sway force is larger than the surge force, the latter often causes high loading in mooring lines because of the lower surge damping. The disruption that passing ships cause to moored ships can often be reduced by paying close attention to the vessels' mooring. Alternative measures are reducing passing ship speeds (which is not always possible as a minimum speed may be required to maintain maneuverability), greater passing distances, or deepening the channel and berth area. As the sizes and speeds of vessels have increased over the years, so have the interaction forces.

Quite logically, the interaction forces on the moored ship increase as the lateral passing distance and UKC decrease and the size and speed of the passing vessel increase (Talstra and Bliek, 2014). Experiments have shown that the forces on a moored ship due to a passing ship are proportional to the passing speed squared, provided the speeds are relatively low. The forces can be significantly larger for larger Froude numbers as the forces significantly divert from the square law assumption roughly for a depth-related Froude number $\mathrm{Fr}_{\mathrm{h}}>0.25$ (Van Der Molen et al., 2011).

Encyclopedia of Maritime and Offshore Engineering, online $\odot 2017$ John Wiley \& Sons, Ltd.

This article is @ 2017 John Wiley \& Sons, Ltd.

DOI: 10.1002/9781118476406.emoe006

Also published in the Encyclopedia of Maritime and Offshore Engineering (print edition) ISBN: 978-1-118-47635-2 

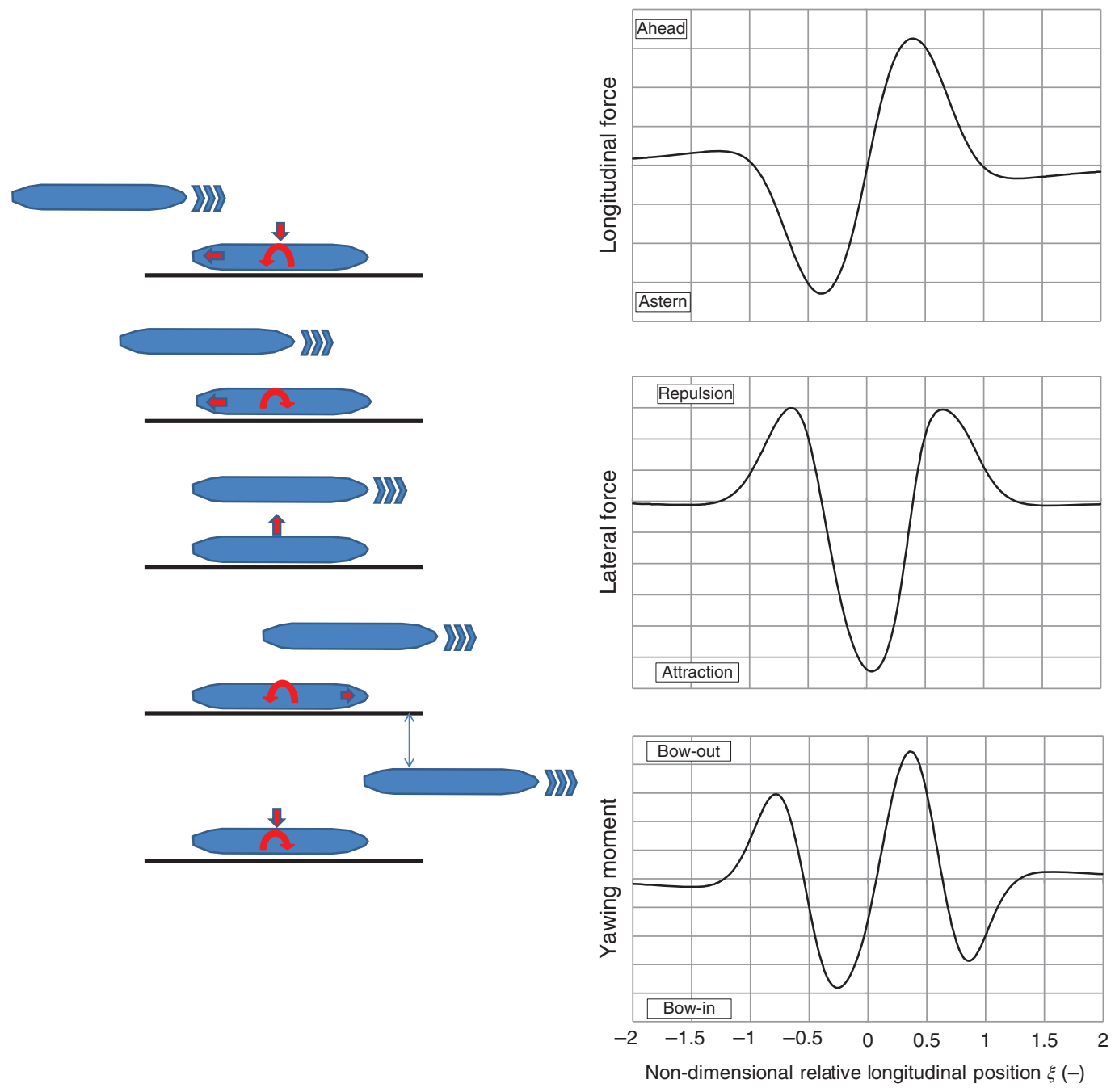

Figure 14. Graphical indication of the interaction forces on a moored ship due to a passing ship (results from ROPES software).

\subsection{Lightering and underway replenishment}

Ship-to-ship transfer of oil or gas is commonly defined as a lightering operation, which usually involves two vessels that are distinctively different in size. The larger ship is referred as a ship to be lightered (STBL), while the smaller ship is named a lightering or a service ship. The operation can be performed in sheltered or open and deep waters. For operations that take place in sheltered waters, the two involved ships are mostly at anchor and moored together, and this situation will not be discussed; attention is paid to lightering operations that involve two ships advancing in parallel side by side at low forward speeds. The situation is similar to a replenishment at sea (RAS) operation, or UNREP, carried out by warships.
The maneuvering requirements for the lightering ship change as she passes through different stages. The lightering ship usually starts her approach from behind, on the starboard side of the STBL. In order to accomplish the lightering operation, that is, the abeam transfer position, the lightering ship must match the speed and heading of the STBL, and the transversal and longitudinal distance relative to this ship has to be monitored continuously. Once the mentioned requirements are fulfilled, both advancing ships are moored together, by using fenders that ensure a lateral separation of up to $5 \mathrm{~m}$ in diameter (Skejic and Berg, 2009).

Potentially hazardous collision situations may develop because of the presence of hydrodynamic interaction between loads when two ships operate in close proximity.

Encyclopedia of Maritime and Offshore Engineering, online (๐ 2017 John Wiley \& Sons, Ltd.

This article is (C) 2017 John Wiley \& Sons, Ltd.

DOI: 10.1002/9781118476406.emoe006

Also published in the Encyclopedia of Maritime and Offshore Engineering (print edition) ISBN: 978-1-118-47635-2 

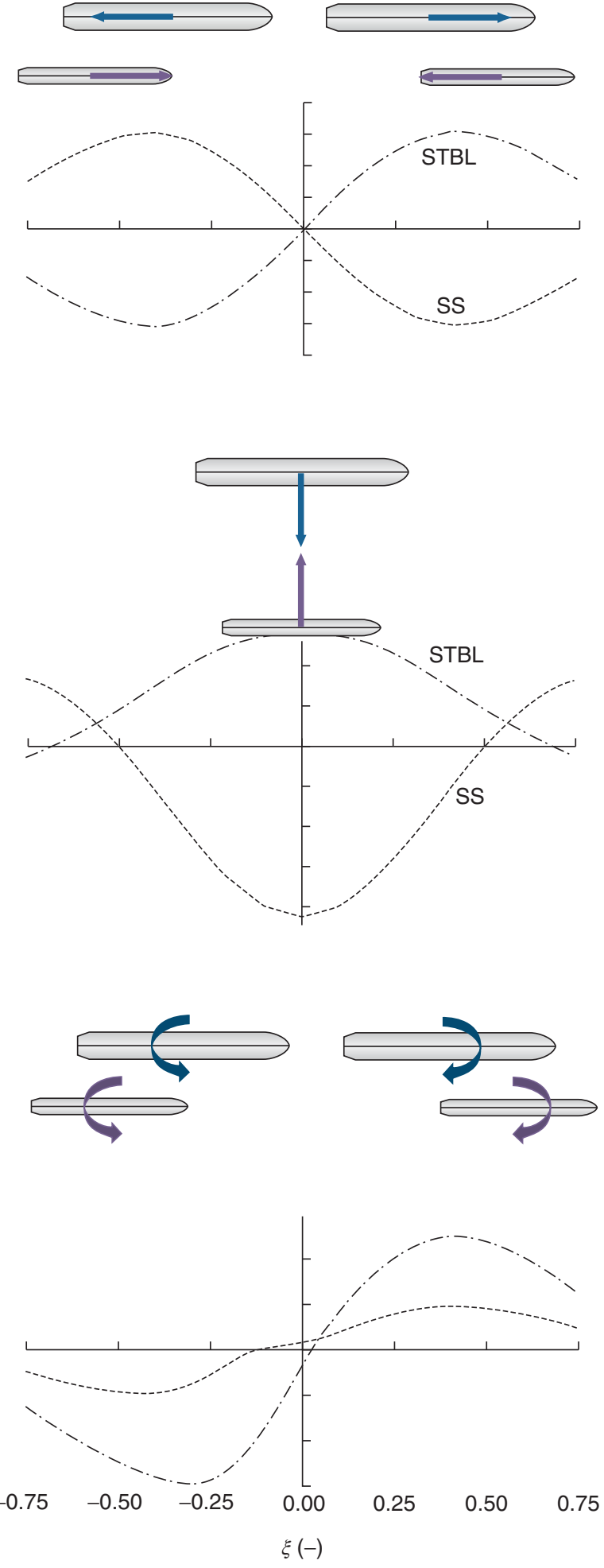

Figure 15. Interaction forces during lightering maneuver as a function of relative longitudinal position of both ships (based on Lataire et al., 2012): longitudinal force (up), lateral force (middle), and yawing moment (down). (Reproduced with permission from Lataire et al., 2012. (C) Elsevier, 2012.)
The interaction forces depend on speed, lateral separation, and relative longitudinal position, as indicated in Figure 15.

\subsection{Tug-ship interaction}

Due to their tasks, tugs have to come close to the ships they assist and sometimes at relatively high speeds, which implies that the interaction forces can be high. To avoid accidents, a good understanding of the interactions between ships and tugs is therefore important for both ship and tug operators (Dand, 1975).

The tug is generally much smaller than the ship it is assisting and while a given depth of water may be deep for the tug, it may well be shallow for the ship. This means that the ship will have a large interactive effect on the tug and the tug will have virtually no effect on the ship (Dand, 1995). In particular, a sway force, a surge force, and a yawing moment will be induced due to the asymmetry of the flow. As usual, these interaction forces will intensify if the flow becomes more two-dimensional (Geerts et al., 2011).

Figure 16 shows diagrammatically the sort of interaction forces and moments conventionally powered (and steered) tugs will typically experience when they come alongside. When the tug is near the stern of the ship, an increase in its velocity may occur due to the flow velocity from the aft of the ship. In close proximity to the ship hull, a low pressure starts moving the tug in the ship's direction. For ships in ballast condition, or ships having particular overhanging stern, the tug risks damage to its hull or superstructure. Going forward and near the hull, the tug experiences an important suction force in the direction of the ship hull and a bow-out yaw moment. When the tug is attracted by the ship, it is in general difficult to recover her course. When the tug is further forward near the side of the bow, she enters high-pressure area and the bow-out yaw moment is growing, which must be compensated by the appropriate use of the rudder and propeller. Finally, when the tug is near the bow, a strong sway force acting on the stern brings the tug to the front and under the bow with the risk of capsizing. This has caught a number of conventional tugs unawares over the years with disastrous consequences, largely due to the sudden changes in the interaction forces and moments acting on the vessel as it alters its fore and aft position alongside the bigger ship. Such variations are larger if the assisted ship has pronounced shoulders (Dand, 1995).

The tug may approach safely in the vicinity of midships where the longitudinal interaction force helps in station-keeping. Some areas near the bow and stern are best avoided because the control that the rudder exerts adds to, rather than subtracts from, the effects of interaction. 


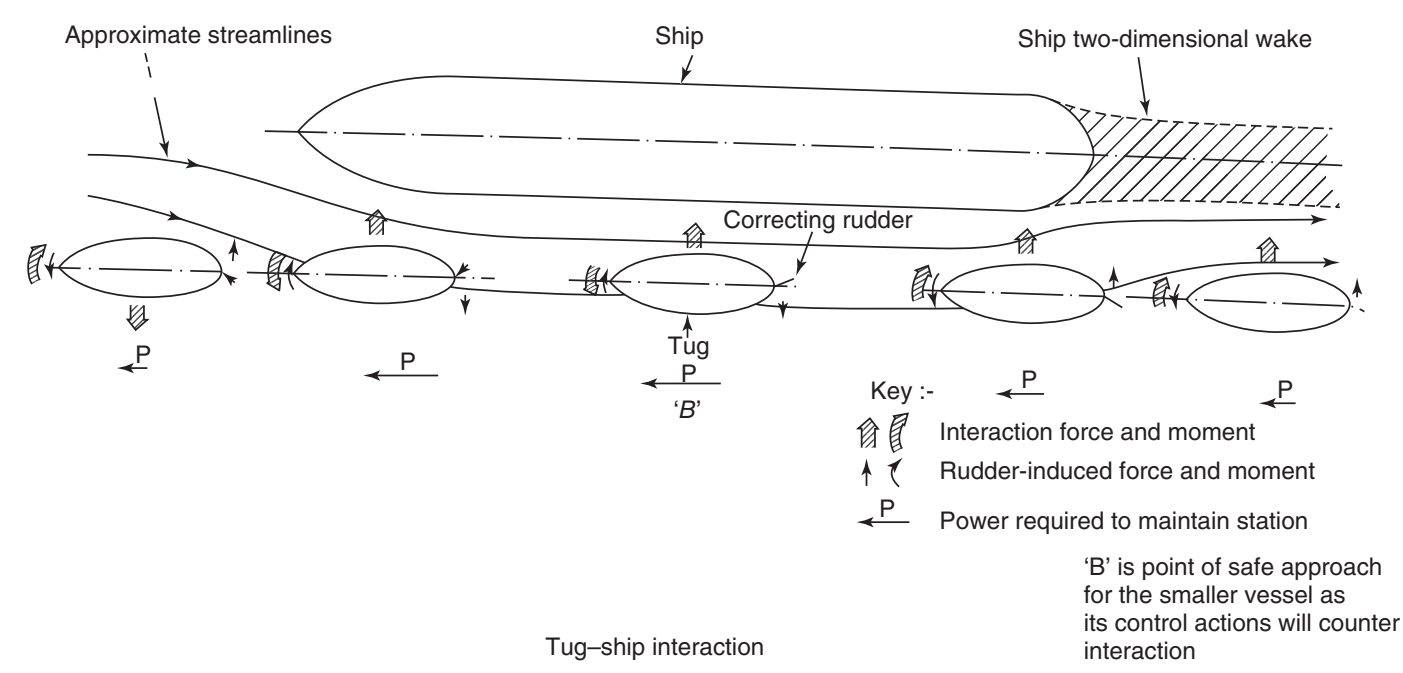

Figure 16. Interaction forces on tugs. (Reproduced with permission from Dand, 1995. ๑ BMI Ltd, 1995.)

\section{NOMENCLATURE}

$a, b \quad$ coefficients in weight distribution function $w(y, z)$

$a_{\mathrm{H}} \quad$ multiplication factor for $Y_{\mathrm{R}}$ to obtain

rudder-induced lateral force acting on the hull

$B \quad$ ship beam (m)

$\mathrm{d} 2 \mathrm{~b}$ non-dimensional distance to bank parameter

$\mathrm{Fr}_{\mathrm{h}} \quad$ depth-related Froude number

$g \quad$ acceleration of gravity $\left(\mathrm{m} / \mathrm{s}^{2}\right)$

$h \quad$ water depth (m)

$h_{1} \quad$ depth of water layer (in muddy areas) or water depth on flooded bank (m)

$h_{\text {avg }} \quad$ average water depth (m)

$k$ bank slope factor

$L \quad$ ship length (m)

$L_{\mathrm{PP}} \quad$ length between perpendiculars (m)

$m \quad$ ship mass $(\mathrm{kg})$ or blockage factor

$m_{\text {eq }} \quad$ equivalent blockage factor

$N \quad$ yawing moment $\left(\mathrm{kg} \mathrm{m}^{2} / \mathrm{s}^{2}\right)$

$N_{\dot{r}} \quad$ added moment of inertia for yaw $\left(\mathrm{kg} \mathrm{m}^{2}\right)$

$r \quad$ yawing rate $(1 / \mathrm{s})$

$\dot{r} \quad$ yaw acceleration $\left(1 / \mathrm{s}^{2}\right)$

$T \quad \operatorname{draft}(\mathrm{m})$

$u \quad$ longitudinal ship speed component $(\mathrm{m} / \mathrm{s})$

$\dot{u} \quad$ ship acceleration in longitudinal direction $\left(\mathrm{m} / \mathrm{s}^{2}\right)$

$U_{\text {crit }} \quad$ critical speed for navigation above fluid mud, as defined in Equation $4(\mathrm{~m} / \mathrm{s})$

UKC under keel clearance

UKCi under keel clearance with respect to the water-mud interface

$v \quad$ lateral ship speed component $(\mathrm{m} / \mathrm{s})$

$\dot{v} \quad$ ship acceleration in lateral direction $\left(\mathrm{m} / \mathrm{s}^{2}\right)$

$V \quad$ ship speed $(\mathrm{m} / \mathrm{s})$
$V_{\text {crit }} \quad$ critical speed in a canal $(\mathrm{m} / \mathrm{s})$

$w(y, z) \quad$ weight distribution function for calculating distance to bank parameter $\mathrm{d} 2 \mathrm{~b}$

$Y \quad$ lateral force $(\mathrm{N})$

$y_{\mathrm{B} 3} \quad$ non-dimensional ship-bank distance parameter

$y_{\mathrm{P}} \quad$ lateral distance at port side from ship's centerline to bank at bottom level (m)

$y_{p 3} \quad$ distance from ship's centerline to bank at port side at half draft $(\mathrm{m})$

$Y_{\mathrm{R}} \quad$ lateral force component (in ship's coordinate system) acting on rudder (N)

$y_{\mathrm{S}} \quad$ lateral distance at starboard side from ship centerline to bank at bottom level (m)

$y_{s 3} \quad$ distance from ship's centerline to bank at starboard side at half draft $(\mathrm{m})$

$Y_{\dot{v}} \quad$ added mass for sway $(\mathrm{kg})$

$\beta \quad$ drift angle $\left({ }^{\circ}\right)$

$\chi \quad$ integral of weight distribution function $w(y, z)$

over a specified domain mentioned in subscript

$\eta_{0} \quad$ non-dimensional ship-bank distance at free surface

non-dimensional ship-bank distance at bottom overall propeller efficiency

water density $\left(\mathrm{kg} / \mathrm{m}^{3}\right)$

mud density $\left(\mathrm{kg} / \mathrm{m}^{3}\right)$

non-dimensional stagger (longitudinal distance between ships on parallel courses)

$\propto \quad$ time derivative of $\alpha(1 / \mathrm{s})$

ITTC International Towing Tank Conference

PIANC The World Association for Waterborne Transport Infrastructure (originally Permanent International Association of Navigation Congresses)

Encyclopedia of Maritime and Offshore Engineering, online (๐ 2017 John Wiley \& Sons, Ltd.

This article is @ 2017 John Wiley \& Sons, Ltd.

DOI: 10.1002/9781118476406.emoe006

Also published in the Encyclopedia of Maritime and Offshore Engineering (print edition) ISBN: 978-1-118-47635-2 
RAS replenishment at sea

STBL ship to be lightered

TEU twenty feet equivalent unit

UNREP underway replenishment

\section{GLOSSARY}

Bank effect Forces and moments acting on a ship due to a motion that has a mainly parallel orientation with respect to a bank.

Blockage Ratio between the cross-sectional area of a ship and the cross-sectional area of a canal.

Critical (of a ship in a waterway) maximum speed speed for which a steady solution for the sinkage and the return flow can be found.

Critical speed

Froude number

Lightering

Nautical bottom

Ship-ship interaction

(in channels with muddy bottoms) maximum speed at which a rising of the water-mud interface under a ship navigating above the mud layer is possible.

Speed of the ship made non-dimensional by division by the square root of the gravitational acceleration multiplied with a characteristic dimension (usually ship length; water depth in case of depth-related Froude number).

Operation during which cargo is transferred between two ships.

The level where physical characteristics of the bottom reach a critical limit beyond which contact with a ship's keel causes either damage or unacceptable effects on controllability and maneuverability.

Hydrodynamic forces and moments induced by the relative speed between two ships, especially when the ship's courses are (nearly) parallel.

Under-keel Vertical distance between a ship's keel and clear- the bottom of the navigation area.
Ch'ng, P.W., Doctors, L.J., and Renilson, M.R. (1993) A method of calculating the ship-bank interaction forces and moments in restricted water. International Shipbuilding Progress, 40 (412), $7-23$.

Crane, C.L. (1979) Maneuvering trials of a 278 000-DWT tanker in shallow and deep waters. SNAME Transactions, 87, 251-283.

Dand, I.W. (1975) Some Aspects of Tug Ship Interaction. Proceedings of the Fourth International Tug Convention, New Orleans, LA, USA, pp. 61-80 (Paper A5).

Dand, I.W. (1995) Interaction. Squat, Interaction, Manoeuvring, The Nautical Institute, Humberside Branch Seminar, pp. 1-20.

Delefortrie, G. (2007) Manoeuvring behaviour of container vessels in muddy navigation areas. Ph.D. thesis, Ghent University.

Delefortrie, G., Vantorre, M., and Eloot, K. (2005) Modelling navigation in muddy areas through captive model tests. Journal of Marine Science and Technology, 10 (4), 188-202.

Eloot, K. (2006) Selection, experimental determination and evaluation of a mathematical model for ship manoeuvring in shallow water. Ph.D. Thesis, Ghent University. ISBN 90-8578-092-6. XIV, 398 pp.

Eloot, K., Vantorre, M. and Delefortrie, G. (2006) Prediction of Ship Manoeuvrability of an 8000 TEU Containership in Deep and Shallow Water: Mathematical Modelling and Captive Model Testing. Marine Simulation and Ship Manoeuvrability: Proceedings of the International Conference MARSIM 2006, Terschelling, The Netherlands, 25-30 June 2006. pp. M-3-1-M-3-9.

Eloot, K., Verwilligen, J. and Vantorre, M. (2007) A Methodology for Evaluating the Controllability of a Ship Navigating in a Restricted Channel. Hydronav 2007, Seventeenth International Conference on Hydrodynamics in Ship, Polanica Zdrój, Poland, September 2007, pp. 114-125.

Geerts, S., Vantorre, M., Eloot, K., Huijsmans, R. and Fierens, N. (2011) Interaction Forces in Tug Operations. Second International Conference on Ship Manoeuvring in Shallow and Confined Water: Ship to Ship Interaction, Trondheim, Norway, R.I.N.A., pp. $153-163$.

ITTC (2002) The Manoeuvring Committee-Final Report and Recommendations to the 23rd ITTC. Proceedings of the 23rd ITTC, Vol. I, pp. 153-234.

Lataire, E. (2014) Experiment based mathematical modelling of ship-bank interaction. Ph.D. thesis, Ghent University.

Lataire, E. and Vantorre, M. (2008) Ship-Bank Interaction Induced by Irregular Bank Geometries. Proceedings 27th Symposium on Naval Hydrodynamics, Seoul.

Lataire, E., Vantorre, M., Delefortrie, G., and Candries, M. (2012) Mathematical modelling of forces acting on ships during lightering operations. Ocean Engineering, 55, 101-115.

Norrbin, N. (1974) Bank Effects on a Ship Moving through a Short Dredged Channel. 10th ONR Symposium on Naval Hydrodynamics, Cambridge, MA.

Norrbin, N. (1985) Bank clearance and optimal section shape for ship canals. 26th PIANC International Navigation Congress, Brussels, Section 1, Subject 1, pp. 167-178.

PIANC (1992) Capability of ship manoeuvring simulation models for approach channels and fairways in harbours. Report of Working Group no. 20 of Permanent Technical Committee II, Supplement to PIANC Bulletin No. 77, 49 pp. 
PIANC (1997) Approach channels—a guide for design. Supplement to PIANC Bulletin 95, 108 pp.

PIANC (2014) Harbour approach channels design guidelines Maritime Navigation Commission, Report No. 121.

Pinkster, J.A. (2004) The influence of a free surface on passing ship effects. International Shipbuilding Progress, 61 (4), 313-338.

Rijkswaterstaat (2011) Waterline Guidelines 2011. DirectorateGeneral for Public Works and Water Management, 177 pp.

Skejic, R. and Berg, T.E. (2009) Hydrodynamic Interaction Effects during Lightering Operation in Calm Water-Theoretical Aspects. MARSIM '09 Conference, Proceedings, Panama City, Panama, Panama Canal Authority, International Marine Simulator Forum, pp. M-8-1-M-8-9.

Talstra, H. and Bliek, A.J. (2014) Loads on Moored Ships Due to Passing Ships in a Straight Harbour Channel, PIANC World Congress, San Francisco, USA.

Van Der Molen, W., Swiegers, P., Moes, J., and Vantorre, M. (2011) Calculation of Forces on Moored Ships due to Passing Ships. Second International Conference on Ship Manoeuvring in Shallow and Confined Water: Ship to Ship Interaction, Trondheim, Norway, R.I.N.A., pp. 369-374.
Vantorre, M. (1991) Ship Behaviour and Control at Low Speed in Layered Fluids. Proceedings International Symposium on Hydroand Aerodynamics in Marine Engineering (HADMAR), BSHC, Varna.

Vantorre, M., Verzhbitskaya, E., and Laforce, E. (2002) Model test based formulations of ship-ship interaction forces. Ship Technology Research, 49 (3), 124-141.

Vantorre, M., Laforce, E., and Delefortrie, G. (2006) A novel methodology for revision of the nautical bottom, in Seminar: Flanders, a Maritime Region of Knowledge (MAREDFlow) (eds Y. Peeters, N. Fockedey, J. Seys, and J. Mees), Vlaams Instituut voor de Zee (VLIZ), Oostende, pp. 15-34.

Verwilligen, J., Vantorre, M., Delefortrie, G., Kamphuis, J., Meinsma, R., and Van Der Made, K.-J. (2014) Manoeuvrability in Proximity of Nautical Bottom in the Harbour of Delfzijl, PIANC World Congress, San Francisco.

Yasukawa, H. and Kobayashi, E. (1995) Shallow Water Model Experiments on Ship Turning Performance. Mini Symposium on Ship Manoeuvrability, 26 May 1995, Fukuoka, Japan, pp. 71-83. 